\title{
Microsymposia
}

MS.06.3

$$
\text { Acta Cryst. (2011) A67, C33 }
$$

\section{Disorder in organic crystals: modelling and prediction}

Matthew Habgood, Department of Chemistry, University College London, London (UK). E-mail: m.habgood@ucl.ac.uk

While the prediction of ordered molecular crystal structures is an increasingly mature discipline, the prediction of disordered molecular crystals has received little attention. A useful approach has emerged through the examination of crystal energy landscapes for ordered structures. Groups of similar structures with closely-spaced energies indicate the possibility of a disordered crystal structure incorporating elements found within the group. However, 'predictions' made in this way are qualitative and rely to a high degree on human intuition. A quantitative approach to predicting disordered crystals must address the usual challenges of crystal structure prediction: the generation of candidate structures, and the calculation and comparison of accurate energies for those candidates. A method has been devised for calculating energies (and other properties) for organic crystals with binary disorder, using the recently introduced idea of 'symmetryadapted ensembles' and implemented using a modified version of the program SOD [1]. Symmetry-adapted ensembles allow for the calculation of average lattice energies and configurational entropies by summing over many copies of a supercell of the disordered crystal, each representing a different distribution of the disorder elements (e.g. different molecular orientations). Only symmetry-unique distributions are explicitly considered, saving an order of magnitude in computer time. Calculations have been carried out on three systems which were not previously open to modelling. The solid solution of dichloro- and dibromo-benzene[2] has been shown to possess a random distribution of the two species, and the observed miscibility gap has been accounted for by calculation of free energy as a function of composition. $\beta$-caffeine is a more complicated case, with two independent molecules displaying orientational disorder. Modelling of this system has confirmed the stability, largely founded on entropy, of the disordered structure proposed by Enright et al. [3] Finally, it has been shown that crystals of 5-ethynyl uracil (eniluracil[4]) display stable disorder, but only in two crystallographic directions. Hydrogen bonded ribbons enforce order in the third direction.

These examples point to near-symmetries in molecular interactions as a key factor in the appearance of disorder, quantifiable through very similar lattice energies of many different symmetryunique configurations. Disorder may occur in all three dimensions, or deviations from near-symmetry may restrict it to a smaller subset. For example, the near-symmetry of the eniluracil molecule is broken by a carbonyl group paired with a hydrogen atom. The consequence of this is ordering in one direction. A combination of the assessment of near-symmetry, and the existing tools of crystal structure prediction - crystal energy landscape generation for ordered structures, and crystal structure matching - seems to allow for the generation of candidate disordered structures, the stabilities of which can now be calculated.

In conclusion, disordered organic crystal structures can now be modelled, and there is a clear route to techniques for predicting their appearance on a similar footing to ordered structures.

[1] R. Grau-Crespo, S. Hamad, C.R.A. Catlow, N.H. de Leeuw, J.Phys.: Condens. Matter 2007, 19, Art.No.256201. [2] A.P. Bussandri, M.J. Zuriaga, C.A.Martin, J. Phys. Chem. Solids 1998, 59, 201-209. [3] G.D. Enright, V.V. Terskikh, D.H. Brouwer and J.A. Ripmeester, Crystal Growth and Design 2007, 7, 1406-1410. [4] R.C.B. Copley et al, Crystal Growth and Design 2008, 8, 3474-3481.

Keywords: disorder, prediction, computer
MS.06.4

Acta Cryst. (2011) A67, C33

\section{Validating Multiple DFT-D methods for crystal-structure prediction}

Jacco van de Streek, Marcus A. Neumann, Avant-garde Materials Simulation, Freiburg (Germany). E-mail: vandestreek@avmatsim.de

The thus far most successful method for evaluating lattice energies for crystal-structure prediction (CSP) is the dispersion-corrected Density Functional Theory (DFT-D) method as published by Neumann \& Perrin in 2005 [1]. Their DFT-D calculations used the PW91 GGA density functional with plane waves in combination with a newlyparameterised dispersion correction. The lattice energies calculated with this method were good enough to predict all four crystal structures of the four small, neutral molecules of the 2007 CSP Blind Test correctly [2].

In the 2010 CSP Blind Test, however, three new categories of chemical compounds were added, for which the results with the 2005 DFT-D method were no longer satisfactory. Especially the relative lattice energies of crystal structures of a hydrate and a molecular salt were poorly reproduced [3].

Since 2005, progress has been made in several areas. First, a new dispersion correction has recently been published by Grimme et al. [4]. Both dispersion corrections involve density functional dependent parameters that need to be fitted to reference data. Second, more density functionals have become available, including hybrid functionals for plane-wave codes. Third, our in-house software for fitting dispersioncorrection parameters has been rewritten to exploit the much faster hardware that has become available since 2005. And fourth, an improved set of reference data, consisting of very low temperature crystal structures $(<30 \mathrm{~K})$, has been collated for fitting and validation purposes.

The two dispersion corrections were fitted in combination with various density functionals, with the aim of determining the best combination for CSP. The fitting procedure and the results will be discussed. For the most promising DFT-D method, updated energy rankings for the molecular salt and for the hydrate of the 2010 CSP Blind Test will be presented.

[1] M.A. Neumann, M.-A. Perrin, J. Phys. Chem. B 2005, 109, 15531-15541. [2] G.M. Day et al., Acta Cryst. B 2009, 65, 107-125. [3] D.A. Bardwell et al., in preparation. [4] S. Grimme, J. Antony, S. Ehrlich, H. Krieger, J. Chem. Phys. 2010, 132,154104

Keywords: density functional theory, Crystal-structure prediction

\section{MS.06.5}

Acta Cryst. (2011) A67, C33-C34

IsoQuestCSP: analyzing sets of predicted crystal structures and selecting the true structure

René de Gelder, Jan M.M. Smits, Maurits Boeije, Hugo Meekes, and Elias Vlieg, Solid State Chemistry, Radboud University Nijmegen, Institute for Molecules and Materials, Nijmegen, (The Netherlands). E-mail: R.deGelder@science.ru.nl

Crystal structure prediction (CSP) has become a powerful and important tool in the study of polymorphism. At the same time CSP is nowadays an alternative route for structure determination when singlecrystal or powder diffraction methods fail or are not accessible. The success of this route is strongly dependent on the quality of the CSP method but also on the ability to recognize the true structure. 


\section{Microsymposia}

CSP methods generate hundreds or thousands of candidate crystal structures among which the structures that can be found experimentally are hopefully present. Usually the energy and density corresponding to predicted structures are guidelines for identifying polymorphs that are likely to be found. In many cases, however, these properties do not point unambiguously to the true structures.

When the true structure is known the set of predicted structures can be analyzed and verified by comparing the crystal structure data of true and predicted structure. For unknown crystal structures this is of course not possible. The only way to compare the predicted set of structures with reality is to match other experimental data (than crystal structure data) with theoretical data that can be deduced from a crystal structure model.

If an experimental powder diffraction pattern (PXRD) is available, this information can be used to select the true structure by comparing it to the simulated patterns of the predicted structures. A complication in doing so is that force fields often tend to induce (anisotropic) deformations in predicted structures, resulting in deformed simulated powder patterns. Powder pattern comparison is then far from straightforward: the true structure can be "hidden" in the predicted set or wrong structures can be selected by mistake.

The program IsoQuestCSP was developed to deal with this problem. It converts a set of predicted structures to a set of PXRD descriptors, compares an experimental pattern with this PXRD descriptor database and generates similarity matrices for predicted sets (suitable for cluster analysis) in an automated fashion. In this way the predicted structures can be analyzed in terms of structural diversity and candidates can be selected that are identical or close to the true structure.

Crucial to the success of the method is applying crosscorrelation functions in combination with (an)isotropic scaling. ${ }^{1,2}$ The method is demonstrated for sets of predicted amino acid structures.

[1] R. de Gelder, R. Wehrens, J. Hageman J. Comp. Chem. 2001, 22, 273-289. [2] R. de Gelder, IUCr CompComm Newsletter. 2006, 7, 59-69.

Keywords: crystal structure prediction, powder pattern comparison, structure determination

\section{MS.07.1}

Acta Cryst. (2011) A67, C34

Pulsed neutron sources for neutron crystallography: new and future capabilities

Robert McGreevy Science and Technology Facilities Council, ISIS Facility, Chilton,Didcot,OX110QX,(UK). E-mail: robert.mcgreevy@) stfc.ac.uk

The last 5 years have seen a remarkable increase in the capabilities of pulsed neutron sources around the world. Although some smaller, older sources have closed (KENS, IPNS), they have been replaced by larger and more powerful facilities - SNS in the USA [1], MLF at JPARC in Japan [2] and ISIS TS2 in the UK [3]. Within the next ten years there is the prospect of the world's first long pulse neutron source - the ESS in Sweden [4] - coming into operation. This presentation will review these new sources and their capabilities for diffraction studies of a range of materials including crystalline, disordered and large scale structures. It will also review the prospects for future development of both sources and techniques and the challenges that will be faced.

[1] http://neutrons.ornl.gov/facilities/SNS [2] http://j-parc.jp/MatLife/en/index. html [3] http://www.isis.stfc.ac.uk/about-isis/target-station-2 [4] http://essscandinavia.eu

Keywords: neutron, source, diffraction
MS.07.2

Acta Cryst. (2011) A67, C34

Investigation of nanometer structures with soft X-ray FEL radiation at FLASH

Josef Feldhaus Deutsches Elektronensynchrotron DESY, Hamburg (Germany). E-mail: josef.feldhaus@desy.de

FLASH, the free electron laser (FEL) facility at DESY in Hamburg, is the first FEL for the vacuum ultraviolet and soft X-ray region in regular user operation. It is based on a superconducting linear accelerator that produces the high-density, low-emittance electron bunches required for driving the FEL. Since 2005, FLASH provides extremely bright, coherent light pulses which can be as short as 10 femtoseconds. After two upgrades in 2007 and 2009/10, FLASH covers a spectral range from $47-4.1 \mathrm{~nm}$ wavelength, i.e. it reaches the so-called water window which allows investigating biological samples with high contrast in their aqueous environment. These unique beam properties have allowed exploring new fields of science, such as non-linear processes in atoms and molecules, ultrafast electronic and magnetic phenomena in solids, and single-pulse imaging of biological samples or nanostructures.

This contribution gives an overview of the FLASH facility and its user programme, with an emphasis on structural investigations.

Keywords: free_electron_laser, soft_X-rays, X-ray_imaging

\section{MS.07.3}

Acta Cryst. (2011) A67, C34

Linac Coherent Light Source: Status and Plans for Expansion John N. Galayda, representing the LCLS and LCLS-II staff. Work supported in part by the DOE Contract DE-AC02-76SF00515. SLAC National Accelerator Laboratory, Menlo Park, California (USA). Email: galayda@slac.stanford.edu

The Linac Coherent Light Source at SLAC is the first "hard" x-ray free-electron laser, providing $\mathrm{x}$-rays in the spectral range 500-10,000 $\mathrm{eV}$ [1]. Experiment operations began in October 2009. LCLS will provide $\mathrm{x}$-rays to six experiment stations, four of which are already in operation. Two of the four have been used for x-ray imaging of nanocrystals and single cells. Early results are very promising [2], [3]. The Department of Energy has approved a concept for a major expansion of the facility (the LCLS-II Project), to increase capacity and to expand its spectral coverage to $250-13,000 \mathrm{eV}$ [4]. The upper end of the range was chosen to enable MAD techniques for structure determination. The presentation will report latest performance and results from LCLS and latest plans for the LCLS-II Project.

[1] P. Emma, et al., Nature Photonics 4, 641. [2] H.N. Chapman, et al., Nature 470, 73-77. [3] J. Hajdu, et al., Nature 470, 475-476. [4] Linac Coherent Light Source II Conceptual Design Report, https://slacspace.slac.stanford.edu/sites/ slac sci controlled/PublishedLibrary/Controlled\%20Documents/\%5B060003-000-00_LCLSIICDR_Index\%5D.pdf

Keywords: free, electron, laser

\section{MS.07.4}

Acta Cryst. (2011) A67, C34-C35

X-ray layout and radiation properties of the European XFEL Thomas Tschentscher, ${ }^{a}$ Winfried Decking, ${ }^{b}$ Torsten Limberg, ${ }^{b}$ Joachim Pflüger, ${ }^{\text {a }}$ Harald Sinn, ${ }^{a}$ Evgeny A. Schneidmiller, ${ }^{\mathrm{b}}$ Mikhail 